ANTICANCER DRUGS

\section{The fat controller}

The ability to target cells with metastatic potential is of crucial importance in cancer treatment, which is

Among those genes, the authors identified increased expression of CD36, a cell surface receptor involved in lipid uptake why new results from the team led by Aznar Benitah, who has identified a subpopulation of cells with high metastatic potential, are exciting news.

The authors were interested in whether a subpopulation of metastasis-initiating cells exists among the CD44 $4^{\text {bright }}$ population, which has been shown to have the highest tumour-initiating potential in oral squamous cell carcinoma (OSCC). They orthotopically injected dyelabelled cell lines and patient-derived cells (PDCs) from human OSCCs into the oral cavity of immunodeficient mice and observed a

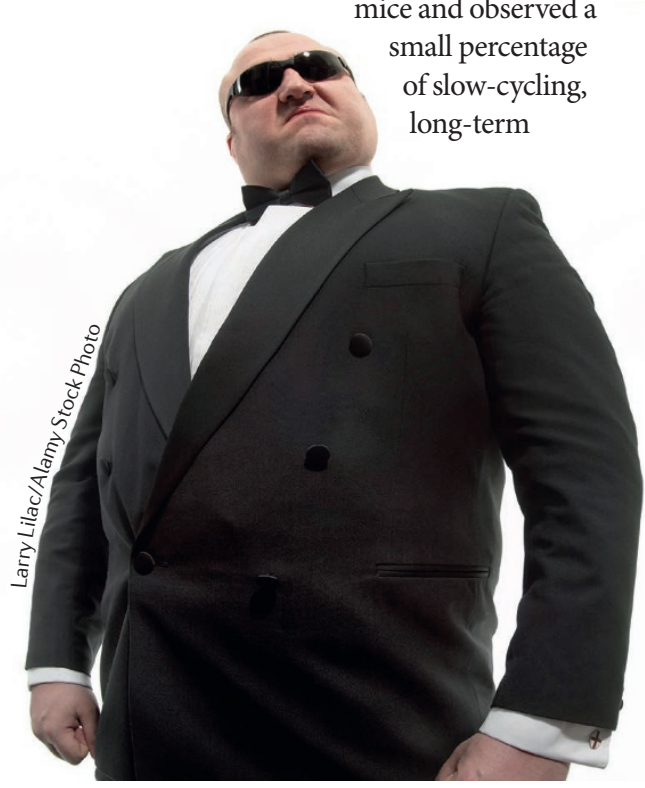

label-retaining cells (LRCs) within oral tumours. Transcriptome analysis of LRCs isolated from tumours from several patients showed differential expression of 69 genes related to metastasis formation and lipid distribution and translocation, compared with the non-LRC population.

Among those genes, the authors identified increased expression of CD36, a cell surface receptor involved in lipid uptake. Overexpression of CD36 in cell lines and PDCs with low metastatic potential greatly increased their potential to metastasize to lymph nodes but did not affect growth of the primary tumours. Depletion of CD36 with short hairpin RNA (shRNA) markedly reduced the penetrance of metastasis to lymph nodes, in some cases up to $100 \%$, whereas it did not have an effect on primary oral lesion growth. Interestingly, the few lymph node metastases that grew from CD36-depleted cells showed a pattern of large cells filled with lipid droplets containing non-metabolized lipids. CD $44^{\text {bright }}$ cells that expressed CD36 also expressed higher levels of three key enzymes involved in fatty acid $\beta$-oxidation (very long-chain specific acyl-CoA dehydrogenase, mitochondrial (ACADVL), medium-chain specific acyl-CoA dehydrogenase, mitochondrial (ACADM) and trifunctional enzyme subunit $\alpha$, mitochondrial (HADHA)) and depletion of long-chain-fatty-acid-CoA ligase 1 (ACSL1), which also activates oxidation of fatty acids, reduced the lymph node metastatic penetrance of both parental OSCC cells and OSCC cells overexpressing CD36. Because lipid $\beta$-oxidation is the most energetically efficient type of oxidation, the authors hypothesized that CD36 ${ }^{+}$ metastatic cells use this pathway to obtain the high amount of energy required for them to colonize and survive at distant sites. Blocking lipid uptake by depleting CD36 resulted in substantial lipotoxicity to the tumour cells owing to accumulation of unmetabolized lipids, but inhibition of other functions of CD36 might also contribute to this anti-metastatic effect.

Mice fed a high-fat diet that were injected with OSCC cells developed more and larger lymph node metastases, with an increase in the percentage of $\mathrm{CD} 6^{+}$cells in both oral and metastatic lesions. Treatment of mice that had been orally inoculated with tumour cells with two anti-CD36 neutralizing antibodies - FA6.152 and JC63.1 - completely inhibited metastasis initiation. Moreover, JC63.1 treatment of mice that had already developed lymph node metastasis reduced the size of lymph node metastases by $80-90 \%$, and resulted in complete remission in $15 \%$ of mice.

These results demonstrate the anti-metastatic effect of blocking CD36, although whether CD36 is a marker of metastatic cells in all tumour types is yet to be tested.

M. Teresa Villanueva, Senior Editor, Nature Reviews Cancer

This article is modified from the original in Nat. Rev. Cancer (http://dx.doi.org/10.1038/nrc.2017.3)

ORIGINAL ARTICLE Pascual, G. et al. Targeting metastasis-initiating cells through the fatty acid receptor CD36. Nature 541, 41-45 (2017) 